\title{
Project Bundling, Liquidity Spillovers, and Capital Market Discipline*
}

\author{
Roman Inderst $^{\dagger} \quad$ Holger M. Müller ${ }^{\ddagger}$
}

January 2000

\begin{abstract}
This paper provides a theory of integration based on the inability of parties to write comprehensive financial contracts. In our model, integration comes with both benefits and costs. On the one hand, integration entails liquidity spillovers from high- to low-return projects, implying that integrated firms have better access to external finance than non-integrated firms. On the other hand, integration leads to the creation of a larger internal capital market, thereby making integrated firms less dependent on the provision of follow-up financing by outside investors. But in a world where financial contracting is incomplete, the threat not to provide follow-up financing may be the only means that investors have to make borrowers repay their debt. By making this threat less effective, integration may aggravate existing financing constraints caused by contractual incompleteness.

Keywords: Incomplete Contracts, Theory of the Firm, Mergers.

JEL Classification Numbers: D32, G32, G34.
\end{abstract}

\footnotetext{
${ }^{*}$ We thank Patrick Bolton, Martin Hellwig, Christian Laux, Benny Moldovanu, and seminar participants at various workshops for helpful comments and suggestions. Financial support from Deutsche Forschungsgemeinschaft, Sonderforschungsbereich 504, is gratefully acknowledged.

${ }_{\dagger}^{\dagger}$ Sonderforschungsbereich 504, University of Mannheim, L13, 15, 68131 Mannheim, Germany. Email: inderst@sfb504.uni-mannheim.de. URL: http://www.vwl.uni-mannheim.de/moldovan/roman.html.

${ }_{\ddagger}^{\ddagger}$ Department of Economics, University of Mannheim, A5, 68131 Mannheim, Germany. Email: hmueller@pool.uni-mannheim.de. URL: http://www.vwl.uni-mannheim.de/hellwig/holger.
} 


\section{Introduction}

Why do some firms merge while others don't? This question, which is closely linked to the question of the boundaries of the firm, has inspired numerous theories and arguments, most of which are based on standard neoclassical concepts like market power and technological efficiency. More recently, however, the focus has shifted toward a contractual view of the firm, the idea being that integration may reduce problems arising from contractual incompleteness. Building on seminal work by Coase (1937) and Williamson (1975, 1985), Grossman and Hart (1986) and Hart and Moore (1990) (henceforth GHM) argue that hold-up problems in a vertical relationship may be alleviated if the firms in question merge. While integration improves the incentives of the controlling firm to make relationship-specific investments, it dulls the incentives of the subordinate firm(s). Integration is optimal if the overall effect is positive.

Along with GHM, this paper provides a theory of integration based on contractual incompleteness. Unlike their model, however, our model is not primarily about vertical integration, nor does it center upon the problem of providing agents with incentives to make relationship-specific investments. Instead, it is based on the inability of parties to write financial contracts ensuring that all positive NPV projects are undertaken. In our model, integration comes with both benefits and costs. On the one hand, integration entails liquidity spillovers from high- to low-return projects, thereby providing integrated firms with better access to external finance than non-integrated firms. The dark side of integration is that, by pooling internal cash flows, integration at least partly shields firms from the disciplinary pressure of the capital market.

In a two-period model, Bolton and Scharfstein (1990) show that in a world where financial contracting is incomplete, the threat not to provide follow-up financing may be the only means that investors have to induce borrowers to repay their debt. While this mitigates the underinvestment problem, it does not solve it completely. In particular, low-return firms are liquidated after the first period even though liquidation is strictly inefficient. Since the investor cannot break even in the second period, he only provides follow-up financing if this allows him to extract a greater repayment in the first period over and above what he can obtain by means of a contract. As for low-return firms, the firm's entire cash flow is verifiable. Consequently, the continuation probability, i.e. the probability of obtaining second-period financing, is zero. At the same time, the highreturn firm's limited liability constraint is slack, implying that the high-return firm has excess first-period liquidity. To skim off the excess liquidity, the investor would have to raise the firm's continuation probability. But this is impossible as the high-return firm already obtains second-period financing with probability one.

If there are two firms, merging the firms (i.e. projects) may alleviate the underin- 
vestment problem even if the firms are ex ante identical. With a certain probability, one of the two projects has a high return while the other has a low return. In this case, if the firms have merged, the investor can skim off some of the high-return project's excess liquidity in exchange for raising the low-return project's continuation probability, thus increasing efficiency. As a result, the merged firm's continuation probability exceeds the average continuation probability that obtains if the two firms remain separate. Incidentally, the outcome cannot be replicated by a contract where, if there is both a low- and a high-return firm, the high-return firm supplies the low-return firm with excess liquidity. The reason is that, since profits are nonverifiable, the high-return firm has an incentive to conceal its type and save on the transfer payment.

Similar to the property rights literature, in our model integration involves a change in the authority structure. If the two firms remain separate, the manager of the lowreturn firm cannot order the manager of the high-return firm to provide the low-return firm with excess liquidity. Also, as was argued above, a contract stipulating such a transfer is impossible due to the non-verifiability of the firms' cash flows. However, if the firms integrate, one of the two managers (or a third party frequently referred to as 'headquarters' in the internal capital markets literature) has the authority to shift funds from one project to another, thus allowing the merged firm to borrow against the projects' combined cash flows. Incidentally, in our model integration involves no change in the underlying information structure as both managers already fully observe each others' cash flows prior to the merger.

There is also a dark side to integration. As integration leads to a pooling of cash flows, the merged firm may be able to finance second-period production with internal funds in cases where the two stand-alone firms had to resort to the capital market. Hence, integration may weaken the investor's termination threat. If this negative effect dominates the spillover effect, integration may no longer be optimal. As is shown, this is the case if the probability that projects have a high return is sufficiently high.

The model also addresses the issue of whether firms should optimally merge with firms whose projects are positively or negatively correlated with their own projects. If the two projects are strongly positively correlated, the probability of having both a highand a low-return project is low. Accordingly, liquidity spillovers are unlikely to occur, and the expected efficiency gain from integration is negligible. Conversely, if the two projects are strongly negatively correlated, the probability of having both a high- and a low-return project is high, and the expected gain from integration is large. Hence, our model offers a rationale for corporate diversification based on the notion that diversified firms have better access to external finance; an explanation that differs from the existing tax and agency cost explanations (e.g. Lewellen 1971; Amihud and Lev 1981; Jensen 
1986; Majd and Myers 1987; Stulz 1990).

In addition to GHM, our paper is related to recent work by Fluck and Lynch (1999). Fluck and Lynch study mergers between firms that are financially constrained and firms that are not financially constrained. For the merger to create synergies, it is crucial that one of the firms is sufficiently profitable to be financed as a stand-alone. By contrast, in our model the question of whether integration is optimal does not hinge on there being an asymmetry between the firms. If both firms are financially constrained, integration may be optimal as it provides the fims with access to external finance. On the other hand, integration may also be optimal if none of the firms is currently financially constrained as it increases the likelihood that external financing will be provided in the future. Moreover, Fluck and Lynch assume exogenous coordination costs to ensure that nonintegration (or divesting) becomes optimal for certain parameter values. By contrast, in our model the costs and benefits of integration are both endogenous.

Our paper also contributes to the growing literature on internal capital markets. Internal capital markets serve to reallocate funds between projects, e.g. to spend the cash flow generated in one division on investment in another division (Williamson 1975; Gertner, Stein, and Scharfstein 1994; Stein 1997). What this suggests is "an interdependence among otherwise completely unrelated investment projects that just happen to be located under the roof of the same company" (Stein 1997, p.112). Similarly, in our model the question of whether a particular project obtains follow-up financing may depend on the performance of the second project. Several recent articles provide evidence in favor of this investment-interdependence hypothesis. For instance, Shin and Stulz (1998) document that the investments of small divisions in diversified firms are strongly related to the cash flows of other divisions. Likewise, Lamont (1997) finds that the investments of non-oil divisions in the U.S. oil industry are linked to the cash flows generated in the firms' core business.

Technically, our paper is related to Mehta (1993) who studies optimal contracting in a principal-agent framework with limited liability and multiple projects. Mehta shows that, as the number of projects goes to infinity, the efficiency loss due to limited liability goes to zero. Similarly, in our model combining individual projects relaxes the firms' limited liability constraints, thus increasing efficiency. Unlike our model, however, Mehta considers a complete contracting framework where output is verifiable.

Finally, our paper is related to, but differs in its focus from, the literature on group lending (Banerjee, Besley, and Guinnane 1994; Besley and Coate 1995; Armendáriz de Aghion 1999). The essential feature of group lending is joint liability, i.e. if any one member of the group does not repay his loan, the entire group is treated as being in default. As in our model, this creates an interdependence among borrowers. Unlike our 
model, however, the group lending literature is primarily concerned with the strategic interaction between borrowers, e.g. free riding, mutual monitoring, or punishment of deviating group members by other group members.

The rest of the paper is organized as follows. Section 2 presents the model. Section 3 contains our main result: if project bundling does not affect the firms' need to return to the capital market for follow-up financing, integration is always optimal. On the other hand, if project bundling reduces the firm's need to return to the capital market, integration is only optimal if the ex ante probability of a high return is sufficiently low. Section 4 shows that this result continues to hold if the firm and the investor can renegotiate both on and off the equilibrium path. Finally, Section 5 considers arbitrary correlations. As is shown, the efficiency gain from integration is inversely related to the projects' correlation coefficient. Section 6 concludes.

\section{The Model}

The model is based on Bolton and Scharfstein (1990). Suppose there are two periods and two firms with no cash or assets. The firms face identical investment opportunities. At the beginning of the first period, each firm can invest $F>0$ and earn an end-of-period return $\pi_{l}<F$ with probability $\theta>0$ and $\pi_{h}>F$ with probability $1-\theta$, where $\pi_{h}>\pi_{l}$. Since $\bar{\pi}:=\theta \pi_{l}+(1-\theta) \pi_{h}>F$, the investment is strictly profitable. The second period is a replica of the first period. For the moment, we assume that projects are uncorrelated both across periods and across firms. In Section 5, we then allow for the case where in any given period the two projects may be correlated with each other.

As a benchmark, consider first the case where the two firms remain separate ('nonintegration). At date 0, a monopolistic investor can make a take-it-or-leave-it offer to each of the firms which the firms accept if the contract provides nonnegative expected value. ${ }^{1}$ The contracting environment is as follows. All relevant variables are observable, but only payments to and from the firms are publicly verifiable. In particular, this implies that neither actual profits nor the firms' investment decisions are verifiable. However, since the project yields at least $\pi_{l}$, the investor, after paying $F$, can always enforce repayment of $\pi_{l}$ as this amount is in the firms' tills regardless of whether they have invested or not. Finally, any profit not paid out at date 1 must remain in the firms and cannot be consumed until date 2 .

Suppose for a moment that there is only one period. At date 1, each firm optimally

\footnotetext{
${ }^{1}$ See Bolton and Scharfstein (1990). Both in the original model by Bolton and Scharfstein and the two-firm variant considered here, the qualitative results remain the same if the take-it-or-leave-it offer is made by the firm(s).
} 
reports that profits are low, implying that the investor gets back only $\pi_{l}<F$. Anticipating this, the investor provides no financing. If, however, there are two periods and the investor is needed in the second period, he can threaten to cut off funding at date 1, thus extracting more than $\pi_{l}$ in the first period. Formally, the assumption that the investor is needed in the second period amounts to assuming that

$$
\pi_{h}-\pi_{l}<F
$$

Incidentally, since $\pi_{l}<F$, the investor's threat to cut off funding is credible.

The investor's contract-design problem can be analyzed as a direct revelation game where the investor pays each firm $F$ dollars at date 0 and the firms each make a report $\hat{s} \in S:=\{l, h\}$ at date $1 .^{2}$ If a firm announces $\hat{s}$, it indicates that its first-period profits are $r(\hat{s})$, where $r(l):=\pi_{l}$ and $r(h):=\pi_{h}$. Based on this report, the firm then pays $R^{1}(\hat{s})$ dollars at date 1 and $R^{2}(\hat{s})$ dollars at date 2 , and the investor pays the firm $F$ dollars with probability $\beta(\hat{s})$ at date 1 to finance second-period production. Note that allowing for a second report at date 2 is pointless as each firm would always report the type corresponding to the lowest possible second-period payment $R^{2}$. Hence, the investor's problem is to find values $\beta(s), R^{1}(s)$, and $R^{2}(s)$ that maximize expected profits subject to the first- and second-period limited liability constraints $R^{1}(s) \leq r(s)$ and $R^{2}(s) \leq r(s)-R^{1}(s)+\pi_{l}$, where $s$ is a firm's true type, as well as the usual truthtelling and individual rationality constraints. Formally, the investor solves

$$
\begin{aligned}
& \max _{\beta(s), R^{1}(s), R^{2}(s)}-F+\theta\left[R^{1}(l)+\beta(l)\left(R^{2}(l)-F\right)\right] \\
& +(1-\theta)\left[R^{1}(h)+\beta(h)\left(R^{2}(h)-F\right)\right]
\end{aligned}
$$

s.t.

$$
\begin{gathered}
r(s)-R^{1}(s)+\beta(s)\left[\bar{\pi}-R^{2}(s)\right] \\
\geq r(s)-R^{1}(\hat{s})+\beta(\hat{s})\left[\bar{\pi}-R^{2}(\hat{s})\right] \text { for all } s, \hat{s} \in S, \\
R^{1}(s) \leq r(s)
\end{gathered}
$$

and

$$
R^{2}(s) \leq r(s)-R^{1}(s)+\pi_{l} \text { for all } s \in S .
$$

The individual rationality constraints have been omitted as they are implied by the limited liability constraints (2)-(3). ${ }^{3}$

\footnotetext{
${ }^{2}$ In principle, a firm's report could condition both on its own type and the other firm's type. As is shown at the end of this section, however, extending the message space does not change results.

${ }^{3}$ Truthtelling can alternatively be ensured by setting $R^{1}(\hat{s})>r(s)$, which makes it physically impossible for type $s$ to mimick type $\hat{s}$. In the case of upwards incentive compatibility, the solution by Bolton and Scharfstein (1990) satisfies both this condition and (1).
} 
As reports depend only on the firms' own types, the optimal contract under nonintegration is the same as in Bolton and Scharfstein (1990). Accordingly, the optimal contract is $\beta(l)=0, \beta(h)=1, R^{1}(l)=R^{2}(h)=\pi_{l}$, and $R^{1}(h)=\bar{\pi}$. Note that, although the optimal contract is not unique, any solution must have $\beta(l)=0, \beta(h)=1$, and $R^{1}(l)=\pi_{l}$. Inserting the optimal contract in the investor's objective function yields

$$
-F+\pi_{l}+(1-\theta)(\bar{\pi}-F)
$$

which implies that the investor invests at date 0 if and only if

$$
F \leq \bar{\pi}-\frac{\bar{\pi}-\pi_{l}}{2-\theta}
$$

The above solution involves two types of inefficiencies. First, if $\bar{\pi}-\left(\bar{\pi}-\pi_{l}\right) /(2-\theta)<$ $F<\bar{\pi}$, the investor does not invest although the project has a strictly positive expected value. Second, if the investor invests, the ex ante expected welfare loss from inefficient liquidation at date 1 is $\theta(\bar{\pi}-F)$. Note, however, that although liquidation is strictly inefficient, there will be no renegotiation on the equilibrium path because the maximum amount that the investor can assure in the second period is $\pi_{l}<F$.

It remains to show that the investor cannot improve upon the above solution by introducing reports that condition both on a firm's own type and the other firm's type. As is well known, if projects are correlated and sufficiently large penalties are possible, reports that condition on the entire state of nature typically yield the first-best (Crémer and McLean 1985, 1988; Johnson, Pratt, and Zeckhauser 1990). In the present case, the firms can fully observe each others' cash flows, implying that the first-best can even be reached if the projects are uncorrelated. However, due to the non-verifiability of first-period wealth, the investor cannot impose penalties over and above what the firms voluntarily announce in their reports, viz. $R^{1}(\hat{s})$. Given this restriction, it is immediate that allowing for an extended message space yields no improvement.

\section{Liquidity Spillovers and Capital Market Discipline}

\subsection{Integration}

Consider now the case where the two firms have merged ('integration'). At date 1, the merged firm can be either of type $(l, l),(l, h)$, or $(h, h)$. The corresponding first-period cash flows are $r(l, l):=2 \pi_{l}$ with probability $\theta^{2}, r(l, h):=\pi_{l}+\pi_{h}$ with probability $2 \theta(1-\theta)$, and $r(h, h):=2 \pi_{h}$ with probability $(1-\theta)^{2}$. Without loss of generality,

\footnotetext{
${ }^{4} \mathrm{~A}$ more subtle issue arises if we allow for renegotiation off the equilibrium path. Off-the-equilibrium path renegotiation is considered in Section 4.
} 
we restrict attention to contracts where the investor pays $2 F$ with probability $\beta(\hat{s})$ at date 1 , and the merged firm pays $R^{1}(\hat{s})$ at date 1 and $R^{2}(\hat{s})$ at date 2 , where the message space is now $\widetilde{S}:=\{(l, l),(l, h),(h, h)\}$. In principle, more general contracts are conceivable, e.g. where the investor randomizes over payments that are different from 0 and $2 F$. As is shown in Appendix A, however, allowing for an extended contracting space leads to the same results.

While we continue to assume that $\pi_{h}-\pi_{l}<F$, we now allow for the possibility that the merged firm can finance one (but only one) second-period project without borrowing new capital at date 1 . Formally, we shall distinguish between the following two cases: if $2\left(\pi_{h}-\pi_{l}\right)<F$, the investor is always needed for the merged firm to operate in the second period ('no self-financing'). On the other hand, if $2\left(\pi_{h}-\pi_{l}\right) \geq F$, the merged firm can finance exactly one second-period project with internal funds if and only if both first-period returns are high ('self-financing'). For expositional clarity, we deal with the two cases in two separate subsections.

\subsection{No Self-Financing}

Under 'no self-financing', the merged firm cannot operate in the second period without borrowing additional capital at date 1. Formally, this amounts to assuming that

$$
2\left(\pi_{h}-\pi_{l}\right)<F
$$

The investor's contract-design problem is then

$$
\begin{aligned}
& \max _{\beta(s), R^{1}(s), R^{2}(s)}-2 F+\theta^{2}\left[R^{1}(l, l)+\beta(l, l)\left(R^{2}(l, l)-2 F\right)\right] \\
& +2 \theta(1-\theta)\left[R^{1}(h, l)+\beta(h, l)\left(R^{2}(h, l)-2 F\right)\right] \\
& +(1-\theta)^{2}\left[R^{1}(h, h)+\beta(h, h)\left(R^{2}(h, h)-2 F\right)\right]
\end{aligned}
$$

s.t.

$$
\begin{gathered}
r(s)-R^{1}(s)+\beta(s)\left[2 \bar{\pi}-R^{2}(s)\right] \\
\geq r(s)-R^{1}(\hat{s})+\beta(\hat{s})\left[2 \bar{\pi}-R^{2}(\hat{s})\right] \text { for all } s, \hat{s} \in \widetilde{S} \\
R^{1}(s) \leq r(s),
\end{gathered}
$$

and

$$
R^{2}(s) \leq r(s)-R^{1}(s)+2 \pi_{l} \text { for all } s \in \widetilde{S}
$$

As under non-integration, the individual rationality constraints have been omitted as they are implied by the limited liability constraints (8)-(9). 
Instead of solving the above problem, we consider a relaxed problem where the global incentive compatibility constraints (7) are replaced by the constraints that neither type $(h, h)$ nor type $(h, l)$ has an incentive to mimick type $(l, l)$. In the proof of Proposition 1 , we then show that the solution to this relaxed problem also solves the original problem. In the relaxed problem, the investor solves (6) subject to the limited liability constraints (8)-(9) and the downwards incentive compatibility constraints

$$
r(s)-R^{1}(s)+\beta(s)\left[2 \bar{\pi}-R^{2}(s)\right] \geq r(s)-R^{1}(l, l)+\beta(l, l)\left[2 \bar{\pi}-R^{2}(l, l)\right],
$$

where $s \in\{(h, h),(h, l)\}$. Denote these downwards incentive compatibility constraints by $C(h, h)$ and $C(h, l)$, respectively. The following two lemmas simplify the analysis.

Lemma 1. At any optimum, it must hold that $\beta(l, l)=0$ and $R^{1}(l, l)=2 \pi_{l}$.

Proof. We argue to a contradiction. Suppose $\beta(l, l)>0$, and define $\bar{R}^{1}(l, l):=2 \pi_{l}$ and $\bar{R}^{2}(l, l):=R^{2}(l, l)-2 \pi_{l}+R^{1}(l, l)$. If $\beta(l, l)<1$, replacing $\left(R^{1}(l, l), R^{2}(l, l)\right)$ with $\left(\bar{R}^{1}(l, l), \bar{R}^{2}(l, l)\right)$ strictly increases the investor's expected profit, whereas if $\beta(l, l)=1$, replacing $\left(R^{1}(l, l), R^{2}(l, l)\right)$ with $\left(\bar{R}^{1}(l, l), \bar{R}^{2}(l, l)\right)$ leaves the investor's expected profit unchanged. Moreover, if $C(h, h), C(h, l)$, and the limited liability constraints hold under $R^{1}(l, l)$ and $R^{2}(l, l)$, they also hold under $\bar{R}^{1}(l, l)$ and $\bar{R}^{2}(l, l)$.

From the second-period limited liability constraint for type $(l, l)$, it follows that $\bar{R}_{i}^{2}(l, l)-2 F<0$. On the other hand, since $\bar{\pi}-F>0$ and $\bar{R}_{i}^{2}(l, l) \leq 2 \pi_{l}$, it must be true that $2 \bar{\pi}-\bar{R}_{i}^{2}(l, l)>0$. Accordingly, reducing $\beta(l, l)$ strictly improves the investor's expected profit without violating any of the incentive compatibility constraints, which contradicts the optimality of $\beta(l, l)>0$. Given that $\beta(l, l)=0$ is optimal, the fact that $R^{1}(l, l)=0$ is also optimal is immediate.

Lemma 2. At any optimum, the incentive compatibility constraints $C(h, l)$ and $C(h, h)$ must be binding.

Proof. We argue again to a contradiction. Suppose $C(h, h)$ is slack. If $\beta(h, h)=0$, $C(h, h)$ implies that the first-period limited liability constraint for type $(h, h)$ must also be slack. But this implies that the investor can improve his expected profit by raising $R^{1}(h, h)$ without violating any constraint, contradiction. If $\beta(h, h) \in(0,1)$, the unique optimal payments for type $(h, h)$ are $R^{1}(h, h)=\pi_{l}+\pi_{h}$ and $R^{2}(h, h)=2 \pi_{l}$. Since $R^{1}(l, l)=2 \pi_{l}$ and $\beta(l, l)=0$ by Lemma 1 , however, this violates $C(h, h)$, contradiction. Finally, if $\beta(h, h)=1$, any optimal contract must satisfy $R^{1}(h, h)+R^{2}(h, h)=2 \pi_{h}+2 \pi_{l}$. Since, $2\left(\pi_{h}-\pi_{l}\right)>2\left(\bar{\pi}-\pi_{l}\right)$, this violates again $C(h, h)$, contradiction.

Next, suppose $C(h, l)$ is slack. If $\beta(h, l)=0$, the argument is the same as above. If $\beta(h, l) \in(0,1)$, the unique optimal payments for type $(h, l)$ are $R^{1}(h, l)=\pi_{h}+\pi_{l}$ 
and $R^{2}(h, l)=2 \pi_{l}$. Observe that if $2 \beta(h, l)\left(\bar{\pi}-\pi_{l}\right) \geq \pi_{h}-\pi_{l}$, this contract is indeed incentive compatible. Since $2\left(\pi_{l}-F\right)<0$, however, the investor is strictly better off by reducing $\beta(h, l)$, contradiction. Finally, if $\beta(h, l)=1$, any optimal contract must satisfy $R^{1}(h, l)+R^{2}(h, l)=\pi_{h}+\pi_{l}+2 \pi_{l}$. In particular, this implies that any optimal contract yields the same profit to the investor as the contract where $R^{1}(h, l)=\pi_{h}+\pi_{l}$ and $R^{2}(h, l)=2 \pi_{l}$. As was shown above, however, the investor would then want to decrease $\beta(h, l)$, contradiction.

Lemma 1 establishes that the lowest type $(l, l)$ receives zero rent in equilibrium. Lemma 2 is a standard feature of contracting problems of this sort. Equipped with Lemmas 1 and 2, we can now derive the optimal contract.

Proposition 1. Under 'no self-financing', the following contract is optimal:

1) Type $(l, l): \beta(l, l)=0$ and $R^{1}(l, l)=2 \pi_{l}$.

2) Type $(l, h): \beta(h, l)=1 /[2(1-\theta)], R^{1}(h, l)=\pi_{h}+\pi_{l}$, and $R^{2}(h, l)=2 \pi_{l}$ if $\theta<1 / 2$, and $\beta(h, l)=1, R^{1}(h, l)=2 \bar{\pi}$, and $R^{2}(h, l)=2 \pi_{l}$ if $\theta \geq 1 / 2$.

3) Type $(h, h): \beta(h, h)=1, R^{1}(h, h)=2 \bar{\pi}$, and $R^{2}(h, h)=2 \pi_{l}$.

Proof. Setting $\beta(l, l)=0$ and $R^{1}(l, l)=2 \pi_{l}$ and inserting the binding $C(h, l)$ and $C(h, h)$ constraints in $(6)$, the objective function can be rewritten as

$$
-2\left(\pi_{l}-F\right)+2 \pi_{l}+4 \theta(1-\theta) \beta(h, l)(\bar{\pi}-F)+2(1-\theta)^{2} \beta(h, h)(\bar{\pi}-F) .
$$

By inspection, (10) is strictly increasing in both $\beta(h, l)$ and $\beta(h, h)$, implying that the solution is $\beta(h, l)=\beta(h, h)=1$ if feasible. If $2 \bar{\pi} \leq \pi_{h}+\pi_{l}$, setting $\beta(h, l)=\beta(h, h)=1$ is indeed feasible. The optimal payments $R^{1}(h, l), R^{2}(h, l), R^{1}(h, h)$, and $R^{2}(h, h)$ then follow from $C(h, l), C(h, h)$, and the respective limited liability constraints.

If $2 \bar{\pi}>\pi_{h}+\pi_{l}$, setting $\beta(h, l)=1$ violates either $C(h, l)$ or the second-period limited liability constraint for type $(h, l)$. Hence, $\beta(h, l)<1$. Next, observe that $2 \bar{\pi}>R^{2}(h, l)$. To see this, suppose to the contrary that $2 \bar{\pi} \leq R^{2}(h, l)$. Subtracting the binding $C(h, l)$ constraint from the second-period limited liability constraint for type $(h, l)$ gives

$$
\pi_{h}+\pi_{l} \geq R^{2}(h, l)+\beta(h, l)\left[2 \bar{\pi}-R^{2}(h, l)\right] .
$$

If $2 \bar{\pi}=R^{2}(h, l)$, this violates $2 \bar{\pi}>\pi_{h}+\pi_{l}$, contradiction. Suppose therefore that $2 \bar{\pi}<$ $R^{2}(h, l)$. Solving (11) for $\beta(h, l)$, we have $\beta(h, l) \geq\left[\pi_{h}+\pi_{l}-R^{2}(h, l)\right] /\left[2 \bar{\pi}-R^{2}(h, l)\right]$, which is strictly greater than 1 since $2 \bar{\pi}<R^{2}(h, l)$ and $2 \bar{\pi}>\pi_{h}+\pi_{l}$ imply that $\pi_{h}+\pi_{l}<$ $R^{2}(h, l)$, contradiction. Solving the binding $C(h, l)$ constraint for $\beta(h, l)$, we obtain $\beta(h, l)=\left[R^{1}(h, l)-2 \pi_{l}\right] /\left[2 \bar{\pi}-R^{2}(h, l)\right]$. Moreover, since $2 \bar{\pi}>R^{2}(h, l)$, it holds that 
$\partial \beta(h, l) / \partial R^{1}(h, l)>\partial \beta(h, l) / \partial R^{2}(h, l)>0$, implying that both the first-and secondperiod limited liability constraint for type $(h, l)$ must be binding. Solving the binding limited liability constraints for $R^{1}(h, l)$ and $R^{2}(h, l)$, we have $R^{1}(h, l)=\pi_{h}+\pi_{l}$ and $R^{2}(h, l)=2 \pi_{l}$. Inserting these values in $\beta(h, l)=\left[R^{1}(h, l)-2 \pi_{l}\right] /\left[2 \bar{\pi}-R^{2}(h, l)\right]$, we finally obtain $\beta(h, l)=\left[\pi_{h}-\pi_{l}\right] /\left[2\left(\bar{\pi}-\pi_{l}\right)\right]=1 /[2(1-\theta)]$.

It remains to show that the solution to the relaxed problem also solves the original problem (6)-(9) . Since $C(h, l)$ and $C(h, h)$ are both binding, all other incentive compatibility constraints are also binding, which implies that the solution is globally incentive compatible.

As in the case of non-integration, some of the payments from the firm to the investor are not unique. However, any optimal contract must exhibit the same continuation probabilities and yield the same expected utility to both the firm and the investor as the contract in Proposition 1.

When choosing an optimal contract, the investor faces a tradeoff between efficiency and rent extraction. While continuation is socially efficient, it involves a personal loss for the investor of $2\left(\pi_{l}-F\right)$. The investor is therefore only willing to finance second-period production if doing so allows him to extract a greater repayment in the first period over and above what he can obtain through a contract, viz. $2 \pi_{l}$. As for type $(l, l)$, the entire first-period profit is verifiable. Consequently, type $(l, l)$ 's continuation probability is 0 . By contrast, type $(h, h)$ 's first-period profit is sufficiently high to compensate the investor for the maximum possible second-period loss. Accordingly, type $(h, h)$ 's continuation probability is 1 . As for type $(h, l)$, the maximum first-period repayment that the investor can extract on top of the verifiable amount $2 \pi_{l}$ is $\pi_{h}-\pi_{l}$. To make type $(h, l)$ indifferent between paying $2 \pi_{l}$ and obtaining no follow-up financing, and paying $2 \pi_{l}$ plus $R^{1}(h, l)-2 \pi_{l} \leq \pi_{h}-\pi_{l}$ and obtaining an expected second-period rent of $\beta(h, l) 2\left(\bar{\pi}-\pi_{l}\right)$, the continuation probability and the repayment $R^{1}(h, l)$ must satisfy

$$
\beta(h, l)=\frac{R^{1}(h, l)-2 \pi_{l}}{2\left(\bar{\pi}-\pi_{l}\right)} .
$$

Clearly, if $\theta \geq 1 / 2$, which implies that $\pi_{h}-\pi_{l} \geq 2\left(\bar{\pi}-\pi_{l}\right)$, it is optimal to set $R^{1}(h, l)=$ $2 \bar{\pi}$ and $\beta(h, l)=1$. Conversely, if $\theta<1 / 2$, the first-period limited liability constraint $R^{1}(h, l)-2 \pi_{l} \leq \pi_{h}-\pi_{l}$ is binding. The solution is then $R^{1}(h, l)=\pi_{h}+\pi_{l}$ and

$$
\beta(h, l)=\frac{\pi_{h}-\pi_{l}}{2\left(\bar{\pi}-\pi_{l}\right)}=\frac{1}{2(1-\theta)},
$$

as stated in the proposition.

By inserting the optimal contract in the investor's objective function, we can compare the investor's profit under integration with his profit under non-integration. The result is summarized in the following proposition. 
Proposition 2. If $\theta<1 / 2$, the investor invests at date 0 if and only if $F \leq$ $\bar{\pi}-\left(\bar{\pi}-\pi_{l}\right) /\left(2-\theta+\theta^{2}\right)$. Conversely, if $\theta \geq 1 / 2$, the investor invests at date 0 if and only if $F \leq \bar{\pi}-\left(\bar{\pi}-\pi_{l}\right) /\left(2-\theta^{2}\right)$. In both cases, integration is optimal.

Proof. If $\theta<1 / 2$, the investor's expected profit under the contract in Proposition 1 is $2\left(\pi_{l}-F\right)+2\left[\theta+(1-\theta)^{2}\right](\bar{\pi}-F)$, and if $\theta \geq 1 / 2$, the investor's expected profit is $2\left(\pi_{l}-F\right)+2\left(1-\theta^{2}\right)(\bar{\pi}-F)$. By contrast, the investor's expected profit under nonintegration is $2\left(\pi_{l}-F\right)+(1-\theta) 2(\bar{\pi}-F)$ (cf. (4)), which is strictly less than either of the two profits under integration for all $\theta$. Solving the respective profits for $F$ completes the proof.

Thus, in the absence of self-financing, integration is always optimal. As the proposition shows, integration reduces both types of inefficiencies that are present if the firms remain separate. First, the critical investment level under integration is greater than under non-integration, implying that more projects are financed if they are put together under the roof of a single firm. Second, if the investor invests, the expected welfare loss from inefficient liquidation at date 1 is $\theta^{2} 2(\bar{\pi}-F)$ if $\theta \geq 1 / 2$ and

$$
\left(\theta^{2}+2 \theta(1-\theta)[1-\beta(h, l)]\right) 2(\bar{\pi}-F)=\left(\theta-\theta^{2}\right) 2(\bar{\pi}-F)
$$

if $\theta<1 / 2$. By contrast, the expected welfare loss under non-integration is $2 \theta(\bar{\pi}-F)$, which is strictly higher than either of the two values under integration.

To see why integration improves efficiency, consider the case where one project has a high return and the other has a low return (if both projects have the same return, the repayments and continuation probabilities are the same under integration and nonintegration). If the two firms remain separate, one of them (type $h$ ) is continued while the other (type $l$ ) is terminated. As argued earlier, increasing type l's continuation probability beyond 0 does not pay for the investor as he cannot extract more than the verifiable amount $\pi_{l}$ in the first period. At the same time, however, type $h$ 's firstperiod limited liability constraint is slack. While type $h$ has excess liquidity of $\pi_{h}-\bar{\pi}$, the investor cannot siphon off this excess liquidity as type $h$ 's continuation probability already attains the maximum value of 1 . By contrast, if the two firms integrate, the investor can siphon off some of type $h$ 's excess liquidity in exchange for raising type l's continuation probability. As a result, the firms' average continuation probability is increased from $[\beta(l)+\beta(h)] / 2=1 / 2$ to $\beta(h, l)=1 /[2(1-\theta)]>1 / 2$ if $\theta<1 / 2$ and $\beta(h, l)=1$ if $\theta \geq 1 / 2$, which explains the efficiency gain. Effectively, integration thus involves a liquidity spillover from the high- to the low-return project with the consequence that it is optimal for the investor to raise the firms' average continuation probability, thereby reducing the amount of inefficient liquidation. 
Incidentally, the efficiency gain cannot be replicated by an insurance contract where, if there is both a high- and a low-return firm, the high-return firm transfers excess liquidity to the low-return firm at date 1 . The reason is that, under any such arrangement the incentive-compatibility constraint of the high-return firm is violated, implying that type $h$ prefers to claim that he is type $l$ rather than make the insurance payment. ${ }^{5}$ In order to realize the efficiency gain, the two firms must therefore necessarily merge.

\subsection{Self-Financing}

Under self-financing, the merged firm can finance one of the two second-period projects with internal funds if and only if both first-period returns are high. Formally, this amounts to assuming that

$$
2 F>2\left(\pi_{h}-\pi_{l}\right) \geq F
$$

Given that type $(h, h)$ can finance a second-period project without borrowing additional capital from the investor at date 1 , the contract in Proposition 1 is no longer incentive compatible. If type $(h, h)$ tells the truth, his expected payoff is $2 \pi_{h}-2 \pi_{l}$. By contrast, if type $(h, h)$ mimicks type $(l, l)$, he can use the retained cash of $2 \pi_{h}-2 \pi_{l}$ to finance a second-period project. His expected payoff is then $2 \pi_{h}-2 \pi_{l}+(\bar{\pi}-F)$, which is strictly greater than his expected payoff from truthtelling. To restore incentive compatibility, the investor must raise type $(h, h)$ 's payoff from truthtelling by reducing either $R^{1}(h, h)$ or $R^{2}(h, h)$. Reducing the first-period repayment $R^{1}(h, h)$ raises a new problem, however, as type $(h, l)$ may then want to mimick type $(h, h)$. The contract below carefully avoids this problem by reducing $R^{2}(h, h)$ and setting $R^{1}(h, h)>\pi_{h}+\pi_{l}$, thereby making it physically impossible for type $(h, l)$ to mimick type $(h, h)$.

Proposition 3. Under self-financing, the following contract is optimal:

1) Type $(l, l): \beta(l, l)=0$ and $R^{1}(l, l)=2 \pi_{l}$.

2) Type $(l, h): \beta(h, l)=1 /[2(1-\theta)], R^{1}(h, l)=\pi_{h}+\pi_{l}$, and $R^{2}(h, l)=2 \pi_{l}$ if $\theta<1 / 2$, and $\beta(h, l)=1, R^{1}(h, l)=2 \bar{\pi}$, and $R^{2}(h, l)=2 \pi_{l}$ if $\theta \geq 1 / 2$.

3) Type $(h, h): \beta(h, h)=1, R^{1}(h, h)=2 \pi_{h}$, and $R^{2}(h, h)=\bar{\pi}-2\left(\pi_{h}-\pi_{l}\right)+F$.

Proof. As under 'no self-financing', we again solve a relaxed problem. The corresponding incentive compatibility constraint for type $(h, h)$, which explicitly takes into account the possibility that type $(h, h)$ can finance one or more second-period projects

\footnotetext{
${ }^{5}$ Recall that in the case of non-integration, the incentive compatibility constraint of type $h$ under the optimal contract holds with equality.
} 
with internal funds by mimicking type $(l, l)$, is denoted by $\bar{C}(h, h) .{ }^{6}$ Type $(h, h)$ 's payoff from deviating and mimicking type $(l, l)$ is then as follows:

$$
U^{D}(h, h)= \begin{cases}2 \pi_{h}-R^{1}(l, l)+\beta(l, l)\left[2 \bar{\pi}-R^{2}(l, l)\right] & \text { if } F \leq 2 \pi_{h}-R^{1}(l, l)<2 F \\ +[1-\beta(l, l)](\bar{\pi}-F) & \\ 2 \pi_{h}-R^{1}(l, l)+\beta(l, l)\left[2 \bar{\pi}-R^{2}(l, l)\right] & \text { if } 2 \pi_{h}-R^{1}(l, l) \geq 2 F . \\ +[1-\beta(l, l)] 2(\bar{\pi}-F) & \end{cases}
$$

Given that $R^{1}(l, l) \leq 2 \pi_{l}$, the case where $2 \pi_{h}-R^{1}(l, l)<F$ can be safely ignored as it violates (A.3). Moreover, observe that Lemmas 1 and 2 continue to hold (with $C(h, h)$ being replaced by $\bar{C}(h, h))$. Since $\beta(l, l)=0$ and $R^{1}(l, l)=2 \pi_{l}$ by Lemma 1 , (A.3) implies that $U^{D}(h, h)=2\left(\pi_{h}-\pi_{l}\right)+\bar{\pi}-F$. As in the proof of Lemma 1, the investor's objective function can then be rewritten as

$$
-2\left(\pi_{l}-F\right)+2 \theta(1-\theta) \beta(h, l) 2(\bar{\pi}-F)+(1-\theta)^{2}(2 \beta(h, h)-1)(\bar{\pi}-F) .
$$

As (12) is strictly increasing in both $\beta(h, l)$ and $\beta(h, h)$, the arguments in the proof of Proposition 1 extend to the present proof. In particular, the optimal contracts for types $(l, l)$ and $(h, l)$ are the same as in Proposition 1. Furthermore, we have $\beta(h, h)=1$, which, in conjunction with $\bar{C}(h, h)$, implies that $R^{1}(h, h)=2 \pi_{h}$ and $R^{2}(h, h)=\bar{\pi}+F-$ $2\left(\pi_{h}-\pi_{l}\right)$. To check for the neglected incentive compatibility constraints, note that it is impossible for type $(h, l)$ to repay $R^{1}(h, h)=2 \pi_{h}$ at date 1 .

Both the repayments and the continuation probability for the lowest and the intermediate type are the same as under 'no self-financing'. What has changed, however, is the repayment for the highest type. Type $(h, h)$ 's payoff is now $\bar{\pi}-F+2\left(\pi_{h}-\pi_{l}\right)$, which is strictly greater than his payoff of $2\left(\pi_{h}-\pi_{l}\right)$ under 'no self-financing'. As the continuation probability of type $(h, h)$ remains the same, this implies that the investor's profit is reduced by $\bar{\pi}-F$. Thus, allowing for the possibility of self-financing causes a shift in profits from the investor to the firm.

As under 'no self-financing', the payments from the firm to the investor are not unique. However, any optimal contract must exhibit the same continuation probabilities and yield the same expected utility to both the firm and the investor as the contract in Proposition 3. Inserting the optimal contract in the investor's objective function and solving for $F$ provides us with the following proposition.

\footnotetext{
${ }^{6}$ We ignore the possibility of on-the-equilibrium-path self-financing. As the solution to the relaxed problem does not allow for this possibility, the restriction is without loss of generality. In Appendix A, however, we consider a more general contracting environment which also allows for the possibility of on-the-equilibrium-path self-financing.
} 
Proposition 4. If $\theta<\sqrt{2}-1$, the investor invests at date 0 if and only if $F \leq$ $\bar{\pi}-\left(\bar{\pi}-\pi_{l}\right) /(2-\theta)$. If $\theta \in[\sqrt{2}-1,1 / 2)$, the investor invests at date 0 if and only if $F \leq \bar{\pi}-\left(\bar{\pi}-\pi_{l}\right) /\left(1+\theta+(1-\theta)^{2} / 2\right)$. Finally, if $\theta \geq 1 / 2$, the investor invests at date 0 if and only if $F \leq \bar{\pi}-\left(\bar{\pi}-\pi_{l}\right) /\left(1+2 \theta(1-\theta)+(1-\theta)^{2} / 2\right)$.

If $\theta<\sqrt{2}-1$, non-integration is optimal. Conversely, if $\theta \geq \sqrt{2}-1$, integration is optimal.

Proof. If $\theta<1 / 2$, the investor's expected profit under the optimal contract in Proposition 3 is $2\left(\pi_{l}-F\right)+2\left(\theta+(1-\theta)^{2} / 2\right)(\bar{\pi}-F)$, and if $\theta \geq 1 / 2$, the investor's expected profit under the optimal contract is $2\left(\pi_{l}-F\right)+2\left(2 \theta(1-\theta)+(1-\theta)^{2} / 2\right)(\bar{\pi}-F)$. By contrast, the investor's expected profit if the two firms remain separate is $2\left(\pi_{l}-F\right)+$ $(1-\theta) 2(\bar{\pi}-F)$, which is strictly less than his profit under integration if and only if $\theta \geq \sqrt{2}-1$. Solving the respective profits for $F$ completes the proof.

As the proposition shows, integration may no longer be optimal if it allows firms to finance second-period production without having to return to the capital market. In a world where financial contracting is incomplete, the threat not to provide follow-up financing (the 'termination threat') may be the only effective instrument that investors have to make firms repay their debt. Without this instrument, investors may be unwilling to provide any financing at all. By reducing the firms' exposure to the disciplinary sanctions of investors, integration may thus aggravate financing constraints caused by contractual incompleteness. In Proposition 4, this is reflected in a decrease in the critical investment level compared with that in Proposition 2.

The decrease in the critical investment level goes hand in hand with a decrease in investor profits. To ensure that type $(h, h)$ does not mimick type $(l, l)$, the investor must raise type $(h, h)$ 's equilibrium payoff compared with that under 'no self-financing'. In fact, type $(h, h)$ 's equilibrium payoff is now higher than twice the equilibrium payoff obtained by type $h$ under non-integration. Accordingly, if the ex ante probability of type $(h, h)$ grows sufficiently large, integration becomes too costly for the investor. In the present context, this is the case if $\theta<\sqrt{2}-1$.

\section{Off-the-Equilibrium-Path Renegotiation}

\subsection{Non-Integration}

As remarked earlier, there is no scope for on-the-equilibrium-path renegotiation as the investor necessarily makes a loss in the second period. If the parties can renegotiate off the equilibrium path, however, the "optimal" contract in Proposition 1 is no longer 
incentive compatible. To see this, suppose type $h$ deviates and mimicks type $l$. In that case, the firm is liquidated even though continuation would yield an efficiency gain of $\bar{\pi}-F$. To realize this gain, the firm would only need additional funds of $F-\left(\pi_{h}-\pi_{l}\right)<\pi_{l}$, implying that it is mutually beneficial for the parties to replace the original contract with a new contract stipulating a payment of $F-\left(\pi_{h}-\pi_{l}\right)$ from the investor to the firm at date 1 and a repayment of $F-\left(\pi_{h}-\pi_{l}\right) \leq x \leq \pi_{l}$ from the firm to the investor at date 2 . Following Bolton and Scharfstein (1990), we assume that in the renegotiation game the firm and the investor can make a take-it-or-leave-it offer with probability $\alpha$ and $1-\alpha$, respectively. Type $h$ 's payoff from mimicking type $l$ and renegotiating is then

$$
\alpha\left(\bar{\pi}-F+\pi_{h}-\pi_{l}\right)+(1-\alpha)\left(\pi_{h}-\pi_{l}\right)=\pi_{h}-\pi_{l}+\alpha(\bar{\pi}-F),
$$

which is strictly greater than his payoff of $\pi_{h}-\pi_{l}$ from truthtelling. To restore incentive compatibility, the investor must raise type $h$ 's truthtelling payoff by $\alpha(\bar{\pi}-F)$, e.g. by increasing $R^{1}(h)$ from $\bar{\pi}$ to $\pi_{h}$ and reducing $R^{2}(h)$ from $\pi_{l}$ to $\bar{\pi}-\left(\pi_{h}-\pi_{l}\right)-\alpha(\bar{\pi}-F) .^{7}$ We thus have the following proposition.

Proposition 5. Under non-integration and off-the-equilibrium path renegotiation, the following contract is optimal:

1) Type $l: \beta(l)=0$ and $R^{1}(l)=\pi_{l}$.

2) Type $h: \beta(h)=1, R^{1}(h)=\pi_{h}$, and $R^{2}(h)=\bar{\pi}-\left(\pi_{h}-\pi_{l}\right)-\alpha(\bar{\pi}-F)$.

Note that, as renegotiation reduces the investor's expected profit by $(1-\theta) \alpha(\bar{\pi}-F)$, both the critical investment level and total welfare are lower than under full commitment.

\subsection{Integration}

As under non-integration, we would expect that allowing for off-the-equilibrium-path renegotiation lowers investor profits and thereby reduces both the critical investment level and total welfare. The question then is whether the decrease in profits is greater under integration or non-integration. If the decrease in profits is greater under integration, the result that under 'no self-financing' integration is always optimal may no longer hold. On the other hand, if the decrease in profits is greater under non-integration, the result that under self-financing non-integration is optimal for sufficiently small values of $\theta$ may no longer be true. The purpose of this section is to illustrate that both results continue to hold if we allow for off-the-equilibrium-path renegotiation. In what

\footnotetext{
${ }^{7}$ Bolton and Scharfstein (1990, Section Ib) propose an alternative, but payoff-equivalent solution where $R^{2}(h)$ is left unchanged and $R^{1}(h)$ is reduced to $\bar{\pi}-\alpha(\bar{\pi}-F)$. Once again, this shows that the transfers under the optimal contract are not unique.
} 
follows, we restrict attention to the case where $\pi_{h}+\pi_{l}<2 F$. The result, however, is perfectly general and extends to all other parameter values (in total, there are 5 cases to be distinguished). We begin with the optimal contract.

Proposition 6. Define $\hat{\theta}:=1-\alpha\left(\pi_{h}-F\right) /\left[\left(\pi_{h}-\pi_{l}\right)(2-\alpha)\right]$, and suppose $\pi_{h}+$ $\pi_{l}<2 F$. Under integration and off-the-equilibrium-path renegotiation, the following contract is optimal:

1) Type $(l, l): \beta(l, l)=0$ and $R^{1}(l, l)=2 \pi_{l}$.

2) Type $(l, h): \beta(h, l)=\left[\pi_{h}-\pi_{l}+\alpha(\bar{\pi}-F)\right] /\left[2\left(\bar{\pi}-\pi_{l}\right)\right], R^{1}(h, l)=\pi_{h}+\pi_{l}$, and $R^{2}(h, l)=2 \pi_{l}$ if $\theta<\hat{\theta}$, and $\beta(h, l)=1, R^{1}(h, l)=\pi_{h}+\pi_{l}$, and $R^{2}(h, l)=2 \bar{\pi}-\pi_{h}+$ $\pi_{l}-\alpha(\bar{\pi}-F)$ if $\theta \geq \hat{\theta}$.

3) Type $(h, h): \beta(h, h)=1$ and $R^{1}(h, h)=2 \pi_{h}$. Moreover, under (A.2) ('no selffinancing'), type $(h, h)$ receives $R^{2}(h, h)=2\left[\bar{\pi}+\pi_{l}-\pi_{h}-\alpha(\bar{\pi}-F)\right]$, whereas under (A.3) (self-financing), he receives $R^{2}(h, h)=2\left[\bar{\pi}+\pi_{l}-\pi_{h}-\alpha(\bar{\pi}-F)\right]-(1-\alpha)(\bar{\pi}-F)$.

Proof. See Appendix B.

Inserting the optimal contract in the investor's objective function, we can again compare the investor's profit under integration with that under non-integration. The following proposition shows that our results are robust with respect to the possibility of off-the-equilibrium-path renegotiation.

Proposition 7. Suppose $\pi_{h}+\pi_{l}<2 F$. Under ' $n o$ self-financing', integration is always optimal. Under self-financing, there exists a critical value $0<\bar{\theta}<1$ such that for all $\theta<\bar{\theta}$ non-integration is optimal, and for all $\theta \geq \bar{\theta}$ integration is optimal.

Proof. Consider first (A.2) ('no self-financing'). For types $(l, l)$ and $(h, l)$, investor profits are the same under integration and non-integration, whereas for type $(h, l)$, investor profits are strictly greater under integration for all $\theta$. Next, suppose (A.3) holds (self-financing), and consider the case where $\theta \geq \hat{\theta}$, implying that $\beta(h, l)=1$. By inspection, investor profits are greater under integration if and only if $(1-\theta)(1-\alpha)<2 \theta$, which is true for all $\theta \geq \hat{\theta}$. On the other hand, if $\theta<\hat{\theta}$, implying that $\beta(h, l)<1$, investor profits are greater under integration if and only if

$$
(1-\theta)(1-\alpha)(\bar{\pi}-F)<2 \theta \frac{\bar{\pi}-F}{\bar{\pi}-\pi_{l}}\left[\pi_{h}-\bar{\pi}+\alpha(\bar{\pi}-F)\right],
$$

or

$$
\theta \geq \bar{\theta}:=\sqrt{1+\left(1+\frac{\alpha}{1-\alpha} \frac{\pi_{h}-F}{\pi_{h}-\pi_{l}}\right)^{2}}-\left(1+\frac{\alpha}{1-\alpha} \frac{\pi_{h}-F}{\pi_{h}-\pi_{l}}\right) .
$$

Comparing $\bar{\theta}$ with $\hat{\theta}$ yields $\hat{\theta}>\bar{\theta}$, which completes the proof. 


\section{Correlated Projects}

If a firm wants to merge with another firm, should it merge with a firm whose projects are positively or negatively correlated with its own projects? In the following, we try to answer this question by investigating to what extent the efficiency gain from integration (if integration is efficient at all) depends on the underlying correlation coefficient $\rho$. Notice that if $\rho=0$, we are back to Section 3 .

While it is immediate that the optimal contracts under both self-financing and "no self-financing' are the same as in Section 3, allowing for correlation between the projects alters the probabilities of the respective types and thus the investor's expected profit. ${ }^{8}$ Recall that if the projects are uncorrelated, the probability of type $(l, l)$ is $\theta^{2}$, the probability of type $(l, h)$ is $\theta(1-\theta)$, and the probability of type $(h, h)$ is $(1-\theta)^{2}$. If the projects are correlated, the corresponding probabilities are $\theta[1-(1-\rho)(1-\theta)]$ for type $(l, l), 2(1-\rho) \theta(1-\theta)$ for type $(l, h)$, and $(1-\theta)[1-\theta(1-\rho)]$ for type $(h, h) .{ }^{9}$ Given these probabilities, we can derive the counterparts of Propositions 2 and 4 . Consider first the case where (A.2) holds ('no self-financing'). We have the following result.

Proposition 8. Suppose (A.2) holds ('no self-financing'). If $\theta<1 / 2$, the investor invests at date 0 if and only if $F \leq \bar{\pi}-\left(\bar{\pi}-\pi_{l}\right) /\left[2-\theta+(1-\rho) \theta^{2}\right]$. Conversely, if $\theta \geq 1 / 2$, the investor invests if and only if $F \leq \bar{\pi}-\left(\bar{\pi}-\pi_{l}\right) /\left[2-\theta^{2}-\rho \theta(1-\theta)\right]$.

In both cases, integration is optimal for all $\rho<1$. If $\rho=1$, integration and nonintegration are revenue-equivalent.

Proof. If $\theta<1 / 2$, the investor's expected profit under the optimal contract in Proposition 1 is $2\left(\pi_{l}-F\right)+2\left[\theta+(1-\theta)^{2}-\rho \theta^{2}\right](\bar{\pi}-F)$, and if $\theta \geq 1 / 2$, the investor's expected profit is $2\left(\pi_{l}-F\right)+2\left[1-\theta^{2}-\rho \theta(1-\theta)\right](\bar{\pi}-F)$. By contrast, the investor's expected profit if the two firms remain separate is $2\left(\pi_{l}-F\right)+(1-\theta) 2(\bar{\pi}-F)$, which is strictly less than (equal to) either of the two profits under integration if $\rho<1$ (if $\rho=1)$. Solving the respective profits for $F$ completes the proof.

${ }^{8}$ To see why the optimal contracts are identical to Section 3 , note that neither the incentive compatibility constraints nor the limited liability constraints depend on the probabilities of the respective types. It is then straightforward to show that Lemmas 1 and 2 continue to hold. Inserting the results from Lemmas 1 and 2 in the investor's objective function shows that the objective function is increasing in both $\beta(h, l)$ and $\beta(h, h)$ for all $\rho$. The rest is analogous to the proof of Proposition 1.

${ }^{9}$ The proof is straightforward. Denote the random variables associated with the two projects by $X$ and $Y$, respectively. The joint probabilities are then $\omega:=\operatorname{Pr}\left(x=\pi_{l}, y=\pi_{h}\right)=\operatorname{Pr}\left(x=\pi_{h}, y=\pi_{l}\right)$, $\operatorname{Pr}\left(x=y=\pi_{l}\right)=\theta-\omega$, and $\operatorname{Pr}\left(x=y=\pi_{h}\right)=1-\theta-\omega$. Since $\rho:=\operatorname{Cov}(X, Y) / \sigma_{X} \sigma_{Y}$ and $\sigma_{X}=\sigma_{Y}$, we have $\rho=1-\omega / \theta(1-\theta)$. Solving for $\omega$ completes the proof.

Incidentally, as $\omega \leq \min [\theta, 1-\theta]$, we obtain as a lower bound for the correlation coefficient $\underline{\rho}=$ $1-(\min [\theta, 1-\theta]) /[\theta(1-\theta)]$. For each $\rho$, we call all $\theta$ for which $\rho \geq \underline{\rho}(\theta)$ holds feasible. 
Recall from Section 3 that integration only matters with respect to the intermediate type $(h, l)$. If the firm is either of type $(l, l)$ or $(h, h)$, the investor's profit under integration and non-integration is the same. Consider now the extreme case where the two projects are perfectly positively correlated. In this case, the probability of type $(h, l)$ is zero, implying that it makes no difference whether the projects are bundled or not. By contrast, if $\rho<1$ the probability of type $(h, l)$ is non-zero and integration is strictly optimal. The efficiency gain from integration is maximal if the two projects are perfectly negatively correlated, in which case the ex ante probability of type $(h, l)$ is 1 . In Proposition 8, this is reflected in the fact that the critical investment level is strictly decreasing in the correlation coefficient $\rho$.

The result that the efficiency gain from integration is decreasing in the amount of correlation is reminiscent of a similar result in portfolio theory. The reason for the diversification benefit is different, however, since all agents are risk neutral. What matters is solely the probability of having a high- and a low-return project at the same time, in which case liquidity spillovers can materialize.

Consider finally the case where (A.3) holds (self-financing). For the sake of brevity, we only consider the question of whether to integrate or not. The corresponding critical investment levels are easy to derive and listed in the working paper version (Inderst and Müller 1999).

Proposition 9. Suppose (A.3) holds (self-financing). Depending on the correlation coefficient, the comparison between integration and non-integration is as follows.

1) $\rho \in(2 / 3,1]:$ Non-integration is always optimal.

2) $\rho \in(1 / 3,2 / 3]:$ If $\theta<1 /[3(1-\rho)]$ non-integration is optimal, whereas if $\theta \geq$ $1 /[3(1-\rho)]$ integration is optimal.

3) $\rho \in(-1 / 2,1 / 3]:$ If $\theta<\tilde{\theta}:=\left(\rho-2+\sqrt{8+\rho^{2}-8 \rho}\right) /[2(1-\rho)]$ non-integration is optimal, whereas if $\theta \geq \widetilde{\theta}$ integration is optimal.

4) $\rho \in[-1,-1 / 2]:$ Integration is always optimal.

Proof. If $\theta<1 / 2$, the investor's expected profit under the optimal contract in Proposition 3 is $2\left(\pi_{l}-F\right)+[1-\theta+\theta(1-\rho)(1+\theta)](\bar{\pi}-F)$, and if $\theta \geq 1 / 2$, the investor's expected profit under the optimal contract is $2\left(\pi_{l}-F\right)+[1+3 \theta(1-\rho)](1-\theta)(\bar{\pi}-F)$. By contrast, the investor's expected profit if the two firms remain separate is $2\left(\pi_{l}-F\right)+$ $(1-\theta) 2(\bar{\pi}-F)$. Comparing expected profits completes the proof.

Proposition 9 is very intuitive. If the correlation coefficient is close to 1 , the probability of having both a high- and a low-return project is negligible. Consequently, 
the liquidity spillover effect is small and the additional inefficiency due to self-financing dominates (Case 1). In this case, non-integration is always optimal. Conversely, if the correlation coefficient is close to -1 , the liquidity spillover effect is sufficiently large and integration is always optimal (Case 4). ${ }^{10}$ For all other values of $\rho$, there exists a critical value of $\theta(1 /[3(1-\rho)]$ in Case 2 and $\tilde{\theta}$ in Case 3$)$ such that integration is optimal if and only if $\theta$ lies above this value. As is easy to show, the critical value is strictly increasing in $\rho$. Moreover, if $\rho=2 / 3$ the critical value is 1 , and if $\rho=0$ the critical value is $\widetilde{\theta}=\sqrt{2}-1$, which corresponds to the solution in Section 3 .

\section{Concluding Remarks}

This paper presents a theory of integration based on the inability of parties to write comprehensive financial contracts. Integration entails both benefits and costs. On the one hand, integration involves liquidity spillovers from high- to low-return projects ensuring that integrated firms can finance more projects than non-integrated firms. On the other hand, integration, by pooling firms' cash flows, reduces the firms' need to return to the capital market for follow-up financing. But in a world where financial contracting is incomplete, the threat not to provide follow-up financing may be the only instrument that investors have to make firms repay their initial debt. By weakening this threat, integration may thus aggravate already existing financing constraints.

In addition to addressing the question of optimal firm size, the paper also addresses the twin question of optimal scope. Given that a firm wants to merge with another firm, should it merge with a firm whose projects are positively or negatively correlated with its own projects? Interestingly, the answer is that bundling negatively correlated projects is always better even though in our model all agents are risk neutral, the reason being that negative correlation maximizes the likelihood that liquidity spillovers materialize. Similarly, one may ask whether a firm's portfolio should optimally consist of projects belonging to the same or a different risk class, or whether it is better to bundle projects with the same or a different profitability. While neither question has been addressed in the paper, the paper provides an ideal framework to examine these and other questions concerning the composition of firms' project portfolios.

Our paper also suggests that integration may be an optimal response to predation. As pointed out by Bolton and Scharfstein (1990), the investors' commitment to terminate funding if a firm's performance is poor may provide other firms with incentives to lower their financially constrained rival's profits, e.g. by increasing advertising or starting a

\footnotetext{
${ }^{10}$ If $\rho \in[-1,-1 / 2]$, there exist values of $\theta$ for which non-integration is optimal, but they lie outside the feasible range (cf. footnote 10).
} 
price war. If there are two financially constrained firms, our results suggest that merging the two firms may reduce their competitors' incentives to prey as poor performance by a project no longer necessarily leads to the project's termination. In particular, if the second project performs well, it may even be true that both projects are continued with probability one. Hence, our model suggests that multi-project firms such as highly diversified conglomerates have a deeper pocket and are thus less vulnerable to predatory behavior by competitors than stand-alone firms.

\section{Appendix A: General Contracting Environment}

This section shows that the contracts derived in Propositions 1 and 3 remain optimal in a more general contracting environment. We allow for the following contracting space. After the investment of $2 F$ at date 0 , the firm announces a type $s$ at date 1 . With probability $\gamma_{i}(s)$, where $i \in I=\{0,1,2\}$ and $\sum_{i \in I} \gamma_{i}(s)=1$, the investor makes (net) payments $N_{i}^{1}(s)$ at date 1 and $N_{i}^{2}(s)$ at date 2 . These payments are chosen such that it is both feasible and optimal for type $s$ to invest in exactly $i$ second-period projects in case the realization of the random variable is $i$.

Given this setup, the investor's objective function is

$$
\begin{aligned}
-2 F- & \theta^{2} \sum_{i \in I} \gamma_{i}(l, l)\left[N_{i}^{1}(l, l)+N_{i}^{2}(l, l)\right]-2 \theta(1-\theta) \sum_{i \in I} \gamma_{i}(h, l)\left[N_{i}^{1}(h, l)+N_{i}^{2}(h, l)\right] \\
& -(1-\theta)^{2} \sum_{i \in I} \gamma_{i}(h, h)\left[N_{i}^{1}(h, h)+N_{i}^{2}(h, h)\right]
\end{aligned}
$$

As in the main text, we consider a relaxed problem. The first- and second-period limited liability constraints for type $s \in \tilde{S}, L_{i}^{1}(s)$ and $L_{i}^{2}(s)$, are as follows:

- If $\gamma_{0}(s)>0, L_{0}^{1}(s)$ and $L_{0}^{2}(s)$ are given by

$$
\begin{aligned}
r(s)+N_{0}^{1}(s) & \geq 0, \\
r(s)+N_{0}^{1}(s)+N_{0}^{2}(s) & \geq 0 .
\end{aligned}
$$

- If $\gamma_{1}(s)>0, L_{1}^{2}(s)$ and $L_{1}^{2}(s)$ are given by

$$
\begin{aligned}
r(s)+N_{1}^{1}(s)-F & \geq 0, \\
r(s)+N_{1}^{1}(s)+N_{1}^{2}(s)-F+\pi_{l} & \geq 0 .
\end{aligned}
$$

- If $\gamma_{2}(s)>0, L_{2}^{1}(s)$ and $L_{2}^{2}(s)$ are given by

$$
\begin{aligned}
r(s)+N_{2}^{1}(s)-2 F & \geq 0 \\
r(s)+N_{2}^{1}(s)+N_{2}^{2}(s)-2 F+2 \pi_{l} & \geq 0 .
\end{aligned}
$$


As an illustration, consider the case where $i=1$. If $L_{1}^{1}(s)$ and $L_{1}^{2}(s)$ both hold, the firm can invest in one second-period project. Since $\bar{\pi}-F>0$, investment is strictly optimal. The firm can, however, also invest in two second-period projects if it has sufficient funds for both investment at date 1 and repayment at date 2 even in the worst possible state. We ignore these additional constraints (for $i=0,1$ ) for the time being. Later, we will show that they are satisfied by the characterized solution.

As in the main text, we restrict attention to the downwards incentive compatibility constraints for types $(h, h)$ and $(h, l)$ with respect to type $(l, l)$ and show that the neglected incentive compatibility constraints hold under the optimal solution. Denote the payoff which type $s$ obtains on the equilibrium path by $U(s)$. Suppose type $s \in\{(h, l),(h, h)\}$ deviates and announces $(l, l)$. The respective payoff if $i \in I$ is realized is denoted by $U_{i}(s)$. To calculate the payoffs for $i=0,1$, we must first determine how many projects types $(h, h)$ and $(h, l)$ can finance given the specified payments $N_{i}^{1}(l, l)$ and $N_{i}^{2}(l, l)$. Note that an upper bound on the number of projects is given by the resources available at date 1 and by the required repayment at date 2. Consider first $i=1$ and suppose that the deviating type $s$ has sufficient funds to invest in two projects, i.e. $r(s)+N_{1}^{1}(l, l) \geq 2 F$. His second-period limited liability constraint is met if $r(s)+2\left(\pi_{l}-F\right)+N_{1}^{1}(l, l)+N_{1}^{2}(l, l) \geq 0$. To see that this constraint is indeed satisfied, simply insert $L_{1}^{2}(l, l)$. Hence, if type $s$ has sufficient funds to invest in two second-period projects, he will be able to repay $N_{1}^{2}(l, l)$ at date 2 . Consider next $i=0$. We can again use the previous argument to show that, if the deviating type $s$ can invest in one project, he can also repay $N_{0}^{2}(l, l)$ at date 2 . To summarize, we have

$$
\begin{aligned}
& U_{2}(s)=r(s)-2 F+N_{2}^{1}(l, l)+N_{2}^{2}(l, l)+2 \bar{\pi}, \\
& U_{1}(s)= \begin{cases}r(s)-F+\bar{\pi}+N_{1}^{1}(l, l)+N_{1}^{2}(l, l) & \text { if } r(s)+N_{1}^{1}(l, l)-2 F<0 \\
r(s)-2 F+2 \bar{\pi}+N_{1}^{1}(l, l)+N_{1}^{2}(l, l) & \text { otherwise, }\end{cases}
\end{aligned}
$$

and

$U_{0}(s)= \begin{cases}r(s)+N_{0}^{1}(l, l)+N_{0}^{2}(l, l) & \text { if } r(s)+N_{0}^{1}(l, l)<F \\ r(s)+N_{0}^{1}(l, l)+N_{0}^{2}(l, l)+2(\bar{\pi}-F) & \text { if } r(s)+N_{0}^{1}(l, l) \geq 2 F \text { and } r(s)-2 F \\ & +2 \pi_{l}+N_{0}^{1}(l, l)+N_{0}^{2}(l, l) \geq 0 \\ r(s)+N_{0}^{1}(l, l)+N_{0}^{2}(l, l)+\bar{\pi}-F & \text { otherwise. }\end{cases}$

The additional case distinction with regard to $U_{0}(s)$ is due to the fact that, unlike $i=1,2$, it does not generally hold for $i=0$ that a firm investing in two projects can automatically repay $N_{0}^{2}(l, l)$ in all states. We are now in the position to state the incentive compatibility constraint for type $s \in\{(h, l),(h, h)\}$, which is denoted by $\hat{C}(s)$ :

$$
U(s) \geq \sum_{i \in I} \gamma_{i}(l, l) U_{i}(s)
$$


The following two claims are analogous to Lemmas 1-2.

Claim 1. At any optimum, it must hold that $\gamma_{0}(l, l)=1$ and $N_{0}^{1}(l, l)+N_{0}^{2}(l, l)=$ $-2 \pi_{l}$. Moreover, it is optimal to set $N_{0}^{2}(l, l)=-2 \pi_{l}$ and $N_{0}^{1}(l, l)=0$.

Proof. We show that $\gamma_{i}(l, l)>0$ is not optimal for $i=1,2$ by arguing to a contradiction. First, suppose the original contract for $i=0$ is replaced by a contract specifying $\bar{N}_{0}^{1}(l, l)=-2 \pi_{l}$ and $\bar{N}_{0}^{2}(l, l)=0$. Clearly, the new contract satisfies $L_{0}^{t}(l, l)$ for $t=1,2$. Moreover, the investor's profit under the new contract is not smaller than under the old contract (in fact, if $L_{0}^{2}(l, l)$ was previously binding, it is not affected at all). It remains to check whether $\hat{C}(s)$ hold for types $(h, h)$ and $(h, l)$. This is indeed the case if $U_{0}(s)$ does not increase as a result of the replacement. But from the definition of $U_{0}(s)$, it is obvious that the deviator's payoff is non-increasing since the sum of payments at dates 1 and 2 is non-increasing while the funds available at date 1 are decreasing. ${ }^{11}$

Next, consider the contract for $i \neq 0$ assuming that $\gamma_{i}(l, l)>0$. Suppose the original contract is replaced by a contract specifying $\bar{N}_{i}^{1}(l, l)=i F-2 \pi_{l}$ and $\bar{N}_{i}^{2}(l, l)=$ $-i \pi_{l}$. Obviously, the investor's payoff cannot decrease as a result of the replacement. Moreover, $U_{i}(s)$ cannot increase, implying that incentive compatibility is preserved. ${ }^{12}$ Given the new contracts $\left(\bar{N}_{0}^{1}(l, l), \bar{N}_{0}^{2}(l, l)\right)$ and $\left(\bar{N}_{i}^{1}(l, l), \bar{N}_{i}^{2}(l, l)\right)$, it then follows that $i F-\bar{N}_{i}^{2}(l, l)<0$ and $U_{i}(s) \geq U_{0}(s)$. Hence, by reducing $\gamma_{i \neq 0}(l, l)$ and increasing $\gamma_{0}(l, l)$, the investor can strictly improve his profit without violating $\hat{C}(s)$, which contradicts the optimality of the original contract where $\gamma_{i}(l, l)>0$ for some $i \neq 0$.

Having proved that $\gamma_{0}(l, l)=1$, it is immediate that $L_{i}^{2}(l, l)$ must be binding, i.e. that $N_{0}^{1}(l, l)+N_{0}^{2}(l, l)=-2 \pi_{l}$. Moreover, the investor is indifferent between payments at date 1 and date 2, while increasing $N_{0}^{1}(l, l)$ at the cost of decreasing $N_{0}^{2}(l, l)$ only relaxes the constraint $\hat{C}(s)$. Hence, there exists a solution specifying $N_{0}^{2}(l, l)=-2 \pi_{l}$ and $N_{0}^{1}(l, l)=0$.

By Claim 1, we have $U_{0}(h, l)=\pi_{h}-\pi_{l}, U_{0}(h, h)=2\left(\pi_{h}-\pi_{l}\right)$ if (A.2) holds ('no self-financing'), and $U_{0}(h, h)=2\left(\pi_{h}-\pi_{l}\right)+\bar{\pi}-F$ if (A.3) holds (self-financing) (note that to derive these expressions, we only need $\gamma_{0}(l, l)=1$ and $N_{0}^{1}(l, l)+N_{0}^{2}(l, l)=-2 \pi_{l}$, which, according to Claim 1, must hold under any solution).

Claim 2. At any optimum, the incentive compatibility constraints $\hat{C}(h, l)$ and $\hat{C}(h, h)$ must be binding.

\footnotetext{
${ }^{11}$ In fact, given $\left(\bar{N}_{0}^{1}(l, l), \bar{N}_{0}^{2}(l, l)\right)$, we obtain $U_{0}(h, l)=\pi_{h}-\pi_{l}$, while for type $(h, h)$ (A.2) implies $U_{0}(h, h)=2\left(\pi_{h}-\pi_{l}\right)$, and (A.3) implies $U_{0}(h, h)=2\left(\pi_{h}-\pi_{l}\right)+\bar{\pi}-F$.

${ }^{12}$ Note that $\bar{N}_{i}^{1}(l, l)=i F-2 \pi_{l}$ and $\bar{N}_{i}^{2}(l, l)=i \pi_{l}$ imply $U_{i}(s)=r(s)-2 \pi_{l}+i\left(\bar{\pi}-\pi_{l}\right)$ for types $(h, h)$ and $(h, l)$.
} 
Proof. Suppose to the contrary that $\hat{C}(s)$ is slack for $s \in\{(h, l),(h, h)\}$. Moreover, suppose that $\gamma_{0}(s)=1$. From $\hat{C}(s)$ it follows that $L_{0}^{1}(s)$ must also be slack, implying that the investor can improve his profit by decreasing $N_{0}^{1}(s)$ without violating any constraint, contradiction. Suppose next that $\gamma_{0}(s)<1$, implying that $\gamma_{i}(s)>0$ for some $i=1,2$, and replace the original contract by a new contract specifying $\bar{N}_{i}^{1}(s)=i F-r(s)$ and $\bar{N}_{i}^{2}(s)=N_{i}^{2}(s)+\left[N_{i}^{1}(s)-\bar{N}_{i}^{1}(s)\right]$. Note that expected payoffs are not affected by this change. In particular, if it was both feasible and optimal for the firm to invest in exactly $i$ projects at date 1 under the original contract, this must also hold under the new contract as the funds available at date 1 are exactly $i F$. Given that $\hat{C}(s)$ is slack, the investor benefits from decreasing $N_{i}^{2}(s)$ until $L_{i}^{2}(s)$ becomes binding (which occurs if $\left.\bar{N}_{i}^{2}(s)=-i \pi_{l}\right)$. If this does not yet generate a contradiction, the investor can strictly improve his profit by decreasing $\gamma_{i}(s)$ and simultaneously increasing $\gamma_{0}(s)$, contradiction (observe that this is possible as $\hat{C}(s)$ is (still) slack).

It follows from Claim 2 that $U(h, l)=\pi_{h}-\pi_{l}, U(h, h)=2\left(\pi_{h}-\pi_{l}\right)$ under 'no selffinancing', and $U(h, h)=2\left(\pi_{h}-\pi_{l}\right)+\bar{\pi}-F$ under self-financing. Inserting the solution for type $(l, l)$ and the binding incentive compatibility constraints in the investor's objective function, it is immediate that the contracts for types $s \in\{(h, l),(h, h)\}$ must be chosen to maximize $(\bar{\pi}-F)\left(\gamma_{1}(s)+2 \gamma_{2}(s)\right)$. The following claim is then obvious.

Claim 3. Under ' $n o$ self-financing', it is optimal to set $\gamma_{2}(h, h)=1, N_{2}^{1}(h, h)=$ $2 F-2 \bar{\pi}$, and $N_{2}^{2}(h, h)=-2 \pi_{l}$. Conversely, under self-financing it is optimal to set $\gamma_{2}(h, h)=1, N_{2}^{1}(h, h)=2 F-2 \pi_{h}$, and $N_{2}^{2}(h, h)=-\bar{\pi}-F+2\left(\pi_{h}-\pi_{l}\right)$. Moreover, if additionally $2 \bar{\pi} \geq \pi_{h}+\pi_{l}$, it is optimal to set $\gamma_{2}(h, l)=1, N_{2}^{1}(h, l)=2 F-2 \bar{\pi}$, and $N_{2}^{2}(h, l)=-2 \pi_{l}$.

It remains to derive the contract for type $(h, l)$ if $2 \bar{\pi}<\pi_{h}+\pi_{l}$.

Claim 4. If $2 \bar{\pi}<\pi_{h}+\pi_{l}$, it is optimal to set $N_{2}^{1}(h, l)=2 F-\pi_{h}-\pi_{l}, N_{2}^{2}(h, l)=$ $-2 \pi_{l}, N_{0}^{1}(h, l)=-\pi_{l}-\pi_{h}, N_{0}^{2}(h, l)=0, \gamma_{2}(h, l)=1 /[2(1-\theta)]$, and $\gamma_{0}(h, l)=(1-$ $2 \theta) /[2(1-\theta)]$.

Proof. First, observe that it cannot be true that $\gamma_{2}(h, l)=1$ as this violates $L_{2}^{2}(h, l)$. Moreover, it is obvious that $\gamma_{0}(h, l)=1$ cannot be optimal either. It therefore remains to check the following three solution candidates.

Candidate $i): \gamma_{2}(h, l)=0$. This immediately implies that $\gamma_{1}(h, l)=1$. Inserting the result in the binding $\hat{C}(h, l)$ constraint yields

$$
N_{1}^{2}(h, l)+N_{1}^{2}(h, l)+\bar{\pi}-F=-2 \pi_{l} .
$$


Hence, we can set $N_{1}^{1}(h, l)=-\pi_{h}-\pi_{l}+F$ and $N_{1}^{2}(h, l)=\pi_{h}-\pi_{l}-\bar{\pi}$.

Candidate $i i): \gamma_{1}(h, l)=0$. By the same arguments as in the proof of Proposition 1 , we have $N_{2}^{1}(h, l)=2 F-\pi_{h}-\pi_{l}, N_{2}^{2}(h, l)=-2 \pi_{l}$, and

$$
\gamma_{2}(h, l)=\frac{\pi_{h}-\pi_{l}}{2\left(\bar{\pi}-\pi_{l}\right)}=\frac{1}{2(1-\theta)}
$$

Moreover, we can set $N_{0}^{1}(h, l)=-\pi_{l}-\pi_{h}$ and $N_{0}^{2}(h, l)=0$.

Candidate iii): $\gamma_{1}(h, l)>0$ and $\gamma_{2}(h, l)>0$. First, notice that $L_{i}^{1}(h, l)$ must be binding, i.e. $N_{i}^{1}(h, l)=i F-\pi_{h}-\pi_{l}$ (if this were not the case, we could shift payments from date 1 to date 2 without affecting expected payoffs.) As $\hat{C}(h, l)$ is binding and $N_{1}^{2}(h, l)>-\pi_{l}$ by $L_{1}^{2}(h, l)$, we obtain

$$
\gamma_{1}(h, l)=\frac{\pi_{h}-\pi_{l}-\gamma_{2}(h, l)\left(N_{2}^{2}(h, l)+2 \bar{\pi}\right)}{\bar{\pi}+N_{1}^{2}(h, l)} .
$$

Note that $0<\gamma_{1}(h, l)<1$. The contract for type $(h, l)$ must then be chosen to maximize $(\bar{\pi}-F)\left(\gamma_{1}(h, l)+2 \gamma_{2}(h, l)\right)$, or

$$
\frac{\pi_{h}-\pi_{l}+\gamma_{2}(h, l)\left[2 N_{1}^{2}(h, l)-2 N_{2}^{2}(h, l)\right]}{\bar{\pi}+N_{1}^{2}(h, l)} .
$$

Since $\gamma_{1}(h, l)$ and $\gamma_{2}(h, l)$ both lie strictly between 0 and 1 , any solution must have $N_{2}^{2}(h, l)=-2 \pi_{l}$, i.e. $L_{2}^{2}(h, l)$ must be binding. Inserting the binding $L_{2}^{2}(h, l)$ constraint in (13) and differentiating with respect to $N_{1}^{2}(h, l)$ shows that the derivative is strictly negative. Hence, it is optimal to set $N_{1}^{2}(h, l)=-\pi_{l}$, in which case $L_{1}^{2}(h, l)$ is binding. It then remains to maximize $(\bar{\pi}-F)\left[\gamma_{1}(h, l)+2 \gamma_{2}(h, l)\right]$ subject to the binding $\hat{C}(h, l)$ constraint, which can be written as $\left[\gamma_{1}(h, l)+2 \gamma_{2}(h, l)\right]\left(\bar{\pi}-\pi_{l}\right)=\pi_{h}-\pi_{l}$. Consequently, the investor is indifferent between shifting probability mass from $i=2$ to $i=1$, which implies that we can restrict attention to candidates i)-ii).

Comparing the investor's profit under i) and ii), we find that profits under candidate ii) are greater than or equal to those under candidate i) if and only if

$$
\theta \leq \frac{\pi_{h}-F}{\pi_{h}-\pi_{l}}
$$

Since $F \leq \bar{\pi}$, the RHS in (14) is bounded from below by $\left(\pi_{h}-\bar{\pi}\right) /\left(\pi_{h}-\pi_{l}\right)=\theta$, implying that (14) indeed holds. Accordingly, the contract specified in ii), which is the same as in Claim 4, is optimal.

Observe that the contracts in Claims 3-4 are equivalent to those in Propositions 1 and 3. It remains to verify that these contracts satisfy the neglected incentive compatibility constraints. First, note that for any $s \in \tilde{S}$ and $i \in I$ with $\gamma_{i}(s)>0$, the unique 
optimal solution is to invest in exactly $i$ projects. Second, if $2 \bar{\pi}<\pi_{h}+\pi_{l}$ and type $(h, h)$ mimics type $(h, l)$, the firm cannot continue at date 1 without additional funding from the investor due to $2 \pi_{h}+N_{0}^{1}(h, l)=\pi_{h}-\pi_{l}$ and (A.1). Consequently, type $(h, h)$ has no incentive to mimic type $(h, l)$. Finally, it is obvious that all upwards incentive compatibility constraints also hold.

\section{Appendix B: Proof of Proposition 6}

As in Section 3, we solve a relaxed program which only considers the downwards incentive compatibility constraints with respect to type $(l, l)$. Unlike Section 3, however, we must now also take into account the possibility that the parties can renegotiate both on and off the equilibrium path. With regard to on-the-equilibrium-path renegotiation, we can safely restrict ourselves to renegotiation-proof contracts. With regard to offthe-equilibrium-path renegotiation, however, this is not possible. Given the contracting space, renegotiation takes place if under the original contract the firm receives no secondperiod funding. In this case, we follow Bolton and Scharfstein (1990) and assume that the firm and the investor can make a take-it-or-leave-it offer with probability $\alpha$ and $1-\alpha$, respectively. The new contract may now specify payments at dates 1 and 2 which trigger investment in either zero, one, or two projects. Of course, the new contract must also satisfy the firm's first- and second-period limited liability constraints.

To set up the investor's maximization problem, denote by $\left(R^{1}(s), R^{2}(s), \beta(s)\right)$ the contract which is chosen by type $s$ on the equilibrium path at date 1 . First, we determine the equilibrium utilities of both parties on and off the equilibrium path. Suppose type $s$ chooses the contract intended for type $\hat{s}$ (in case $s \neq \hat{s}$, we are only interested in $s=(h, l)$ or $s=(h, h)$, and $\hat{s}=(l, l))$. If $\beta(\hat{s})=1$, renegotiation is not an issue. If $\beta(\hat{s})<1$, denote the firm's (continuation) payoff in case it receives no funding under the old contract by $\bar{U}(s, \hat{s})$. Similarly, denote the investor's (continuation) payoff by $\bar{V}(s, \hat{s})$. To derive these payoffs, we must distinguish between six cases.

Case 1: $r(s)-R^{1}(\hat{s}) \geq 2 F$. In this case, type $s$ can continue both projects without additional funding, which yields $\bar{U}(s, \hat{s})=r(s)-R^{1}(\hat{s})+2(\bar{\pi}-F)$ and $\bar{V}(s, \hat{s})=R^{1}(\hat{s})$.

Case 2: $F \leq r(s)-R^{1}(\hat{s})<2 F$ and $r(s)-R^{1}(\hat{s}) \geq 2\left(F-\pi_{l}\right)$. While the firm can only pursue a single project with internal funds, it can always obtain funding for the second project. To see this, observe that continuing both projects requires only $2 F-$ $\left[r(s)-R^{1}(\hat{s})\right]$, which is less than or equal to $2 \pi_{l}$. Hence, there exists a contract satisfying both the firm's first- and second-period limited liability constraints while allowing the investor to break even. If the firm makes the take-it-or-leave-it offer, the continuation payoffs, which shall be denoted by $\bar{U}^{F}(s, \hat{s})$ and $\bar{V}^{F}(s, \hat{s})$, are $\bar{U}^{F}(s, \hat{s})=r(s)-R^{1}(\hat{s})+$ 
$2(\bar{\pi}-F)$ and $\bar{V}^{F}(s, \hat{s})=R^{1}(\hat{s})$, respectively. If the investor makes the offer, the issue is somewhat more involved. Straightforward computations show that the maximum that the investor can realize is

$$
\bar{V}^{I}(s, \hat{s})=R^{1}(\hat{s})+\min \left[\bar{\pi}-F, r(s)-R^{1}(\hat{s})-2 F+2 \pi_{l}\right],
$$

whereas the firm's payoff is given by

$$
\bar{U}^{I}(s, \hat{s})= \begin{cases}r(s)-R^{1}(\hat{s})+\bar{\pi}-F & \text { if } r(s)-R^{1}(\hat{s})-2 F+2 \pi_{l} \geq \bar{\pi}-F \\ 2\left(\bar{\pi}-\pi_{l}\right) & \text { otherwise. }\end{cases}
$$

We then have $\bar{U}(s, \hat{s})=\alpha \bar{U}^{F}(s, \hat{s})+(1-\alpha) \bar{U}^{I}(s, \hat{s})$. Derivation of $\bar{V}(s, \hat{s})$ is analogous.

Case 3: $F \leq r(s)-R^{1}(\hat{s})<2 F$ and $r(s)-R^{1}(\hat{s})<2\left(F-\pi_{l}\right)$. In this case, the firm can only continue a single second-period project, for which it already has the necessary resources. Consequently, we have $\bar{U}(s, \hat{s})=r(s)-R^{1}(\hat{s})+\bar{\pi}-F$ and $\bar{V}(s, \hat{s})=R^{1}(\hat{s})$.

Case 4: $r(s)-R^{1}(\hat{s})<F$ and $r(s)-R^{1}(\hat{s}) \geq 2\left(F-\pi_{l}\right)$. While the firm does not have the funds to finance one or even more second-period projects, it can always obtain sufficient funds to continue both projects. As in Case 2, we thus have $\bar{U}^{F}(s, \hat{s})=$ $r(s)-R^{1}(\hat{s})+2(\bar{\pi}-F)$ and $\bar{V}^{F}(s, \hat{s})=R^{1}(\hat{s})$. If the investor makes the take-it-or-leaveit offer, we must distinguish between the case where he continues with one project and where he continues with two projects. If he continues with one project, he realizes

$$
\bar{V}^{I, 1}(s, \hat{s})=R^{1}(\hat{s})+\min \left[\bar{\pi}-F, r(s)-R^{1}(\hat{s})-F+\pi_{l}\right],
$$

whereas if he continues with two projects, his payoff is

$$
\bar{V}^{I, 2}(s, \hat{s})=R^{1}(\hat{s})+\min \left[2(\bar{\pi}-F), r(s)-R^{1}(\hat{s})-2 F+2 \pi_{l}\right] .
$$

To resolve the investor's possible indifference, we assume that if both alternatives yield the same payoff, he continues with both projects. The firm's payoff if one and two second-period projects are continued is then

$$
\bar{U}^{I, 1}(s, \hat{s})= \begin{cases}r(s)-R^{1}(\hat{s}) & \text { if } r(s)-R^{1}(\hat{s})-F+\pi_{l} \geq \bar{\pi}-F \\ \bar{\pi}-\pi_{l} & \text { otherwise }\end{cases}
$$

and

$$
\bar{U}^{I, 2}(s, \hat{s})= \begin{cases}r(s)-R^{1}(\hat{s}) & \text { if } r(s)-R^{1}(\hat{s})-2 F+2 \pi_{l} \geq 2(\bar{\pi}-F) \\ 2\left(\bar{\pi}-\pi_{l}\right) & \text { otherwise, }\end{cases}
$$

respectively.

Case 5: $r(s)-R^{1}(\hat{s})<F$ and $F-\pi_{l} \leq r(s)-R^{1}(\hat{s})<2\left(F-\pi_{l}\right)$. In this case, the firm cannot continue without the investor's aid, and its resources only allow for external 
funding of one second-period project. We thus have $\bar{U}^{F}(s, \hat{s})=r(s)-R^{1}(\hat{s})+\bar{\pi}-F$ and $\bar{V}^{F}(s, \hat{s})=R^{1}(\hat{s})$, while

$$
\bar{V}^{I}(s, \hat{s})=R^{1}(\hat{s})+\min \left[\bar{\pi}-F, r(s)-R^{1}(\hat{s})-F+\pi_{l}\right]
$$

and

$$
\bar{U}^{I}(s, \hat{s})= \begin{cases}r(s)-R^{1}(\hat{s}) & \text { if } r(s)-R^{1}(\hat{s})-F+\pi_{l} \geq \bar{\pi}-F \\ \bar{\pi}-\pi_{l} & \text { otherwise. }\end{cases}
$$

Case 6: $r(s)-R^{1}(\hat{s})<F-\pi_{l}$. As there is no renegotiation, we obtain $\bar{U}^{F}(s, \hat{s})=$ $r(s)-R^{1}(\hat{s})$ and $\bar{V}^{F}(s, \hat{s})=R^{1}(\hat{s})$.

We are now in the position to state the incentive compatibility constraints $\tilde{C}(s)$ for types $s \in\{(h, h),(h, l)\}$ :

$$
\begin{aligned}
& \beta(s)\left[r(s)-R^{1}(s)+2 \bar{\pi}-R^{2}(s)\right]+[1-\beta(s)] \bar{U}(s, s) \\
\geq & \beta(l, l)\left[r(s)-R^{1}(l, l)+2 \bar{\pi}-R^{2}(l, l)\right]+[1-\beta(l, l)] \bar{U}(s,(l, l)) .
\end{aligned}
$$

Using the results from Cases 1-6, we have the following claim (recall that by assumption, $\left.\pi_{h}+\pi_{l}<2 F\right)$.

Claim 1. If $R^{1}(l, l)=2 \pi_{l}$, the firm's continuation payoffs from mimicking type $(l, l)$ and renegotiating are as follows:

i) Type $(h, h): \bar{U}((h, h),(l, l))=2\left(\pi_{h}-\pi_{l}\right)+2 \alpha(\bar{\pi}-F)$ under (A.2) ('no self-financing'), and $\bar{U}((h, h),(l, l))=2\left(\pi_{h}-\pi_{l}\right)+(\alpha+1)(\bar{\pi}-F)$ under (A.3) (self-financing).

ii) Type $(h, l): \bar{U}((h, l),(l, l))=\pi_{h}-\pi_{l}+\alpha(\bar{\pi}-F)$.

Proof. Consider first type $(h, h)$. Suppose (A.2) holds, and set $s=(h, h)$ and $\hat{s}=$ $(l, l)$. Since $2\left(\pi_{h}-\pi_{l}\right)>2\left(F-\pi_{l}\right)$, we are in Case 4 . If the investor makes the offer, his continuation payoffs in the one- and two-project case are $\bar{V}^{I, 1}(s, \hat{s})=\bar{\pi}-F$ and $\bar{V}^{I, 2}(s, \hat{s})=2(\bar{\pi}-F)$, respectively, implying that $\bar{V}^{I, 2}(s, \hat{s})>\bar{V}^{I, 1}(s, \hat{s})$. From this it follows that $\bar{U}(s, \hat{s})=2\left(\pi_{h}-\pi_{l}\right)+2 \alpha(\bar{\pi}-F)$. On the other hand, if (A.3) holds, Case 2 applies since $r(s)-R^{1}(\hat{s})=2\left(\pi_{h}-\pi_{l}\right) \geq F, r(s)-R^{1}(\hat{s})<2 F$, and $2\left(\pi_{h}-\pi_{l}\right)>2\left(F-\pi_{l}\right)$. As $r(s)-R^{1}(\hat{s})-2 F+2 \pi_{l}>\bar{\pi}-F$, it immediately follows that $\bar{U}(s, \hat{s})=2\left(\pi_{h}-\pi_{l}\right)+(\alpha+1)(\bar{\pi}-F)$.

Next, consider type $(h, l)$ and set $s=(h, l)$ and $\hat{s}=(l, l)$. Since $r(s)-R^{1}(\hat{s})=\pi_{h}-\pi_{l}$, $\pi_{h}-\pi_{l}<F$ by (A.1), $\pi_{h}-\pi_{l}>F-\pi_{l}$, and $\pi_{h}-\pi_{l}<2\left(F-\pi_{l}\right)$, Case 5 applies. As $r(s)-R^{1}(\hat{s})-F-\pi_{l}>\bar{\pi}-F$, it follows that $\bar{U}(s, \hat{s})=\pi_{h}-\pi_{l}+\alpha(\bar{\pi}-F)$.

The rest of the proof is analogous to that in Proposition 1 and 3 or Appendix A. We first derive the unique optimal contract for type $(l, l)$. 
Claim 2. At any optimum, it must hold that $\beta(l, l)=0$ and $R^{1}(l, l)=2 \pi_{l}$.

Proof. The proof is similar to that of Lemma 1. We first prove that $\beta(l, l)=0$ by arguing to a contradiction. Suppose therefore that $\beta(l, l)>0$ and adjust $R^{2}(l, l)$ such that the second-period limited liability constraint for type $(l, l)$ is binding. Note that this increases the investor's payoff, while $\tilde{C}(s)$ still holds for types $s \in\{(h, l),(h, h)\}$. Next, set $\bar{R}^{1}(l, l)=2 \pi_{l}$ and $\bar{R}^{2}(l, l)=R^{2}(l, l)-\left(2 \pi_{l}-R^{1}(l, l)\right)$, and define $\Delta R:=2 \pi_{l}-R^{1}(l, l)$. We claim that for $\Delta R>0$, this improves the investor's payoff while $\tilde{C}(s)$ still holds. If $\beta(l, l)=1$, the investor is indifferent. If $\beta(l, l)<1$, however, the investor's payoff increases in states where no second-period funding is provided. Moreover, checking Cases 1-6 confirms that $\bar{V}((l, l),(l, l))$ is non-decreasing. A similar argument holds with respect to $\tilde{C}(s)$, i.e. as the deviating type $s$ must pay the extra amount $\Delta R$ in case the project is terminated, his renegotiation payoff $\bar{U}(s,(l, l))$ is non-increasing.

We can now argue as in Lemma 1 . By decreasing $\beta(l, l)$, the investor's payoff strictly increases without violating $\tilde{C}(s)$ since $\bar{R}^{2}(l, l)<2 F$ by the second-period limited liability constraint for type $(l, l)$, contradiction. It then follows that $R^{1}(l, l)=2 \pi_{l}$.

We proceed as follows. First, we derive the optimal contract for types $(h, h)$ and $(h, l)$ from the class of renegotiation-proof contracts. Thus, if the contract specifies $\beta(s)<1$, this requires that $\bar{U}(s, s)=r(s)-R^{1}(s)$ and $\bar{V}(s, s)=R^{1}(s)$ (this can always be ensured by setting $\left.R^{1}(s)=r(s)\right)$. Second, we show that the restriction to renegotiation-proof contracts is without loss of generality. ${ }^{13}$

The argument is now the same as in Propositions 1 and 3. Accordingly, $\tilde{C}(s)$ is binding and can be inserted in the investor's objective function, which yields the contracts in Proposition 6. As these contracts only solve the relaxed problem, we must check whether the neglected incentive compatibility constraints are satisfied. First, note that since $R^{1}(h, l)=\pi_{h}+\pi_{l}$, the contracts are indeed renegotiation-proof. Moreover, type $(h, l)$ cannot mimic type $(h, h)$, implying that we only need to check whether type $(h, h)$ has an incentive to mimick type $(h, l)$. In this case, type $(h, h)$ 's resources are equal to $\pi_{h}-\pi_{l}$. Two cases must be distinguished. First, suppose (A.2) holds. Since additionally $\pi_{h}+\pi_{l}<2 F$, Case 5 applies. Moreover, $\bar{U}^{I}((h, h),(h, l))=\pi_{h}-\pi_{l}$. Hence, $\bar{U}((h, h),(h, l))$ is given by

$$
\begin{aligned}
& \beta(h, l)\left[\pi_{h}-\pi_{l}+2\left(\bar{\pi}-\pi_{l}\right)\right]+(1-\beta(h, l))\left[\pi_{h}-\pi_{l}+\alpha(\bar{\pi}-F)\right] \\
= & \pi_{h}-\pi_{l}+\beta(h, l) 2\left(\bar{\pi}-\pi_{l}\right)+(1-\beta(h, l)) \alpha(\bar{\pi}-F) .
\end{aligned}
$$

\footnotetext{
${ }^{13}$ As we operate in a restricted contracting environment, the renegotiation-proofness principle does not necessarily apply. It must thus be shown that restricting attention to renegotiation-proof contracts involves no loss of generality.
} 
Inserting $\beta(h, l)=\left[\pi_{h}-\pi_{l}+\alpha(\bar{\pi}-F)\right] / 2\left(\bar{\pi}-\pi_{l}\right)$ yields

$$
2\left(\pi_{h}-\pi_{l}\right)+2 \alpha(\bar{\pi}-F)-\alpha(\bar{\pi}-F) \frac{\pi_{h}-\pi_{l}+\alpha(\bar{\pi}-F)}{2\left(\bar{\pi}-\pi_{l}\right)},
$$

which is bounded from above by type $(h, h)$ 's equilibrium payoff $2\left(\pi_{h}-\pi_{l}\right)+2 \alpha(\bar{\pi}-F)$. On the other hand, if (A.3) holds, Case 3 applies. In this case, incentive compatibility is immediate as $\bar{U}((h, h),(h, l))=\pi_{h}-\pi_{l}$.

It remains to show that restricting attention to renegotiation-proof contracts for types $s \in\{(h, l),(h, h)\}$ is without loss of generality. Suppose to the contrary that the contract for some type $s \in\{(h, l),(h, h)\}$ is renegotiated on the equilibrium path. In this case, we can straightforwardly specify a new contract where $\bar{R}^{1}(s)=r(s), \bar{R}^{2}(s) \leq 2 \pi_{l}$, and $\bar{\beta}(s) \leq 1$. As is easy to verify, this contract earns both type $s$ and the investor the same profit as the original contract and is not renegotiated if $\bar{\beta}(s)<1$.

\section{References}

Amihud, Y., and Lev, B. (1981), "Risk Reduction as a Managerial Motive for Conglomerate Mergers," Bell Journal of Economics 12, 605-617.

Armendáriz de Aghion, B. (1999), "On the Design of a Credit Agreement with Peer Monitoring," Journal of Development Economics 60, 79-104.

Banerjee, A., Besley, T., and Guinnane, T. (1994), "Thy Neighbor's Keeper: The Design of a Credit Cooperative with Theory and Test," Quarterly Journal of Economics 109, 491-515.

Bolton, P., and Scharfstein, D.S. (1990), "A Theory of Predation Based on Agency Problems in Financial Contracting," American Economic Review 80, 93-106.

Besley, T., and Coate, S. (1995), "Group Lending, Repayment Incentives and Social Collateral," Journal of Development Economics 46, 1-18.

Coase, R. (1937), "The Nature of the Firm," Economica 4, 386-405.

Crémer, J., and McLean, R. (1985), "Optimal Selling Strategies under Uncertainty for a Discriminating Monopolist When Demands are Interdependent," Econometrica $53,345-361$.

Crémer, J., and McLean, R. (1988), "Full Extraction of the Surplus in Bayesian and Dominant Strategy Auctions," Econometrica 56, 1247-1258. 
Fluck, S., and Lynch, A.W. (1999), "Why Do Firms Merge and Then Divest? A Theory of Financial Synergy," Journal of Business 72, 319-346.

Gertner, R.H., Scharfstein, D.S., and Stein, J.C. (1994), "Internal versus External Capital Markets," Quarterly Journal of Economics 109, 1211-1230.

Grossman, S.J., and Hart, O.D. (1986), "The Costs and Benefits of Ownership: A Theory of Vertical and Lateral Integration," Journal of Political Economy 94, 691719 .

Hart, O.D., and Moore, J.H. (1990), "Property Rights and the Nature of the Firm," Journal of Political Economy 98, 1119-1158.

Inderst, R., and Müller, H.M. (1999), "Project Bundling, Liquidity Spillovers, and Capital Market Discipline," Discussion Paper 99-89, Sonderforschungsbereich 504, University of Mannheim.

Jensen, M.C. (1986), "Agency Costs of Free Cash Flow, Corporate Finance, and Takeovers," American Economic Review 76, 323-329.

Johnson, S., Pratt, J., and Zeckhauser, R. (1990) "Efficiency Despite Mutually PayoffRelevant Private Information: The Finite Case" Econometrica 58, 873-900.

Lamont, O. (1997), "Cash Flow and Investment: Evidence from Internal Capital Markets," Journal of Finance 52, 83-109.

Lewellen, W.G. (1971), "A Pure Financial Rationale for the Conglomerate Merger," Journal of Finance 26, 521-537.

Majd, S., and Myers, S.C. (1987), "Valuing the Government's Tax Claim on Risky Assets," NBER Working Paper 1553.

Mehta, S.R. (1993), "A Solution to a Class of Agency Problems, with Application to the Question of Limited Liability," Economics Letters 43, 193-197.

Shin, H.-H., and Stulz, R.M. (1998), “Are Internal Capital Markets Efficient?" Quarterly Journal of Economics 113, 531-553.

Stein, J.C. (1997), "Internal Capital Markets and the Competition for Corporate Resources," Journal of Finance 52, 111-133.

Stulz, R.M. (1990), "Managerial Discretion and Optimal Financing Policies," Journal of Financial Economics 26, 3-27. 
Williamson, O.E. (1975), Markets and Hierarchies: Analysis and Antitrust Implications. New York: Free Press.

Williamson, O.E. (1985), The Economic Institutions of Capitalism. New York: Free Press. 